\title{
Three-Dimensional Joint Kinematics in a Canine Elbow Joint with Medial Coronoid Disease before and after Bi-Oblique Dynamic Proximal Ulnar Osteotomy
}

\author{
Thomas Rohwedder $^{1}$ Pia Rebentrost ${ }^{2}$ Peter Böttcher ${ }^{1}$
}

${ }^{1}$ Small Animal Clinic, Freie Universität Berlin, Berlin, Germany

${ }^{2}$ Department of Small Animal Medicine, University of Leipzig Faculty

Address for correspondence Thomas Rohwedder, Dr. med.vet., of Veterinary Medicine, Leipzig, Sachsen, Germany

\begin{abstract}
Keywords

- coronoid process

- kinematography

- elbow

- ulnar osteotomy

- canine

Objective The aim of this study was to report the humeroulnar joint kinematics in a dog with medial coronoid process disease (MCPD) before and after dynamic proximal ulnar osteotomy (DPUO).

Study Design A 15-month-old female Labrador Retriever with advanced MCPD was treated by DPUO and fragment removal. Bi-planar fluoroscopic kinematography of the affected joint was performed before and 12 weeks after DPUO along with computed tomography. Static axial radioulnar incongruence (sRUI), dynamic relative proximodistal radioulnar motion (dynamic RUI), axial humeroulnar rotation, as well as humeroulnar joint contact at the medial coronoid process (MCP) were calculated.

Results Static axial radioulnar incongruence was reduced from 2.3 to $1.5 \mathrm{~mm}$ after DPUO but dynamic RUI remained unchanged $(0.2$ vs. $0.3 \mathrm{~mm})$. Mean humeroulnar rotational amplitude increased from $2.6^{\circ}$ (standard deviation 0.4 ) to $4.5^{\circ}$ (standard deviation 2.0). Joint contact area at the MCP became substantially increased as well as broadly distributed among the MCP following DPUO ( 52.5 vs. $63.0 \% ; p=0.0012$ ). Conclusion Dynamic proximal ulnar osteotomy failed to restore the radioulnar congruence and increased the humeroulnar rotational instability. No effect was observed on dynamic RUI. Nevertheless, joint contact area at the MCP was increased and became more homogeneously distributed, which might explain the beneficial effect of clinical outcome in this case.
\end{abstract}

\section{Introduction}

Developmental elbow disease is a common cause of forelimb lameness especially in young dogs, being bilateral in 25 to $80 \%$ of cases. ${ }^{1-5}$ A permanent developmental mismatch in the radioulnar joint surface, called static axial radioulnar incongruence (sRUI), has been shown to increase load at the medial coronoid process (MCP). ${ }^{6,7}$ Being related to the severity of joint pathology at the medial joint compartment, ${ }^{8}$ sRUI is commonly cited as one factor for the development of medial coronoid process disease (MCPD) ${ }^{3,9}$ Other factors, which have been cited as a potential biomechanical cause of MCPD, are dynamic RUI (dRUI) and humeroulnar rotational instability. ${ }^{10-16}$ Dynamic RUI is defined as an increased motion between the radius and ulna, leading to a temporary incongruence of the radioulnar joint surface during locomotion. Among those, sRUI as well as dRUI have recently been excluded as primary causes of MCPD. ${ }^{10,17,18}$

Dynamic proximal ulnar osteotomy (DPUO) was introduced in the 1990s to correct sRUI and to overall improve joint congruence. ${ }^{19,20}$ Correction of positive sRUI leads to received

April 27, 2019

accepted after revision

September 2, 2019
DOI https://doi.org/

$10.1055 / \mathrm{s}-0039-1698440$. ISSN $2625-2325$. (c) 2019 Georg Thieme Verlag KG Stuttgart · New York
License terms

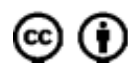


more physiologically balanced joint loads at the medial joint compartment. $^{21,22}$ However, DPUO fails to re-establish radioulnar joint congruence in vivo, while at the same time DPUO ameliorates focal humeroulnar joint contact at the MCP. $^{23}$

We had the unique opportunity to follow a dog with MCPD, with positive sRUI and humeroulnar instability until bony union of a DPUO, using in vivo fluoroscopic kinematography. Our working hypothesis was that DPUO would reduce humeroulnar rotational instability, improve sRUI and would re-establish a homogenously distributed contact pattern at the MCP.

\section{Case Description}

A 15-month-old female Labrador Retriever with a body weight of $27 \mathrm{~kg}$ was presented to the Department of Small Animal Medicine, University of Leipzig, because of unilateral, left sided, grade $2 / 5$, forelimb lameness. ${ }^{24}$ Both forelimbs were held in slight supination and abduction. Pain was elicited on palpation of the left elbow. Otherwise, the dog showed no other clinical abnormalities. Orthogonal radiographs of both elbow joints revealed subtrochlear sclerosis and signs of secondary osteoarthritis at the left elbow joint. Osteoarthritic changes were classified as grade 2 according to the International Elbow Working Group. ${ }^{25}$ Based on these findings, the tentative diagnosis of MCPD was made and confirmed by computed tomography (CT) and arthroscopy.

\section{Advanced Imaging Findings and Surgical Treatment}

Transverse CT with a slice thickness of $1 \mathrm{~mm}$ and an overlapping increment of $0.5 \mathrm{~mm}$ (Philips Brilliance; Philips, the Netherlands) of both elbow joints was performed under general anaesthesia with the dog in dorsal recumbence. Computed tomography showed fragmentation of the MCP at the left side, while the right elbow showed no signs of MCPD (-Fig. 1). Quantification of sRUI using the sphere fitting technique on three-dimensional (3D) renderings of the radioulnar joint cup ${ }^{26}$ provided evidence of a $2.3 \mathrm{~mm}$ positive sRUI in the left elbow (-Fig. 2A).

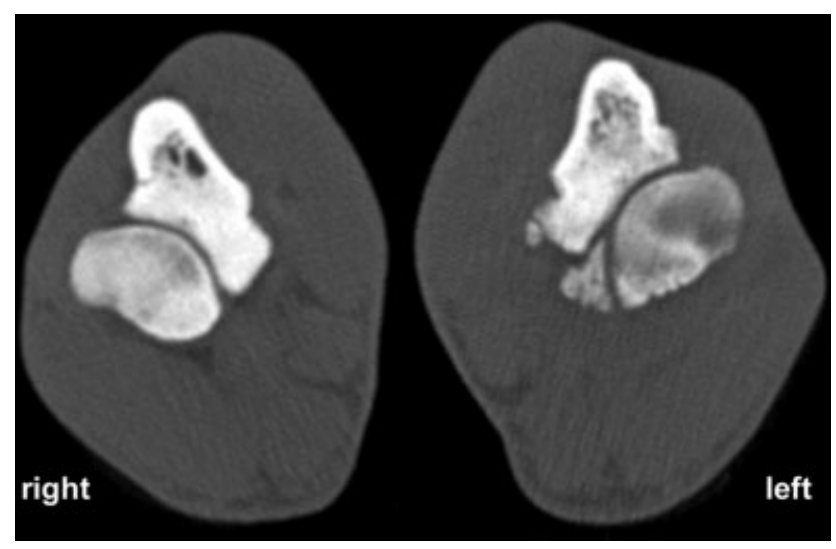

Fig. 1 Preoperative computed tomography scan of both elbow joints; transverse view onto radius and ulna at the level of the medial coronoid process. Right side (sound); left side (affected joint). An $8 \times 5$-mm fragment is present at the medial coronoid process; osteophytes are present at the radius and ulna.

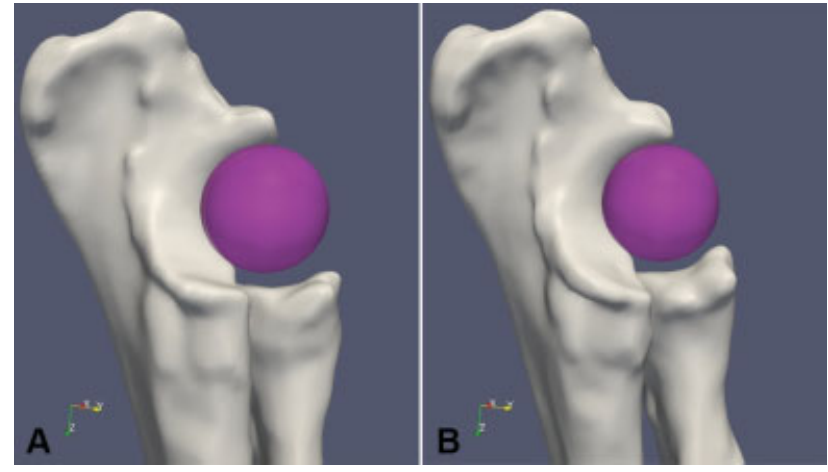

Fig. 2 Sphere fitting technique for estimation of radioulnar incongruence, medial view. (A) Preoperative joint conformation; the sphere fits within the trochlear notch, but loses contact to the radial joint surface due to positive radioulnar step formation. (B) Postoperative joint conformation; the sphere still has no contact with the radial joint surface, but step formation is reduced. Further caudodistal rotation of the proximal ulna segment is visible.

Findings of standard medial arthroscopy of the left elbow using a $1.9 \mathrm{~mm} 30^{\circ}$ fore-oblique scope (Storz Endoskope, Tuttlingen, Germany) matched up with the results of the CT scan, showing a positive radioulnar incongruence, a nondisplaced fragment at the radial incisure of the MCP as well as Modified Outerbridge grade 2 to 3 cartilage lesions at the MCP and grade 3 to 4 lesions on the opposing humeral trochlea ( - Fig. 3A, B). Arthroscopic evaluation of radioulnar incongruence was performed, using a scaled hook probe (Arthrex VetSystems, Munich, Germany). The fragment was removed via arthroscopy and the joint lavaged. Further surgical treatment, such as DPUO, was declined by the owners at that time. The right joint was not approached due to lack of clinical signs.

Five weeks later bi-oblique DPUO, as described by Fitzpatrick, ${ }^{27}$ was performed at the request of the owners ( - Fig. 4), due to ongoing lameness of the left forelimb. A second transverse CT scan of the affected elbow joint was acquired 12 weeks following DPUO when the dog underwent laparoscopic ovariectomy. Quantification of sRUI using the sphere fitting technique on these CT-based 3D bone models showed a radioulnar step of $1.5 \mathrm{~mm}$ (-Fig. 2B). Comparing the preoperative $3 \mathrm{D}$ renderings to the images following DPUO,

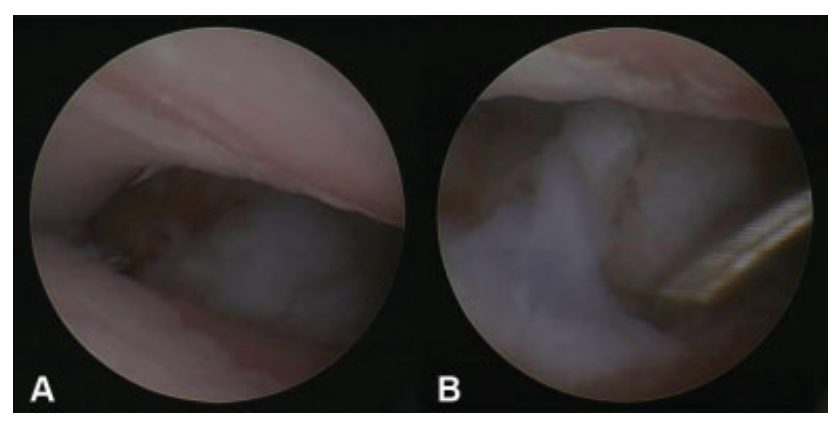

Fig. 3 Arthroscopic examination through standard medial approach. (A) Grade III and IV Outerbridge scale cartilage lesions can be seen at the medial humeral condyle and at the medial coronoid process. (B) View onto the loose fragment of the medial coronoid process after displacement with an arthroscopic hook probe. 

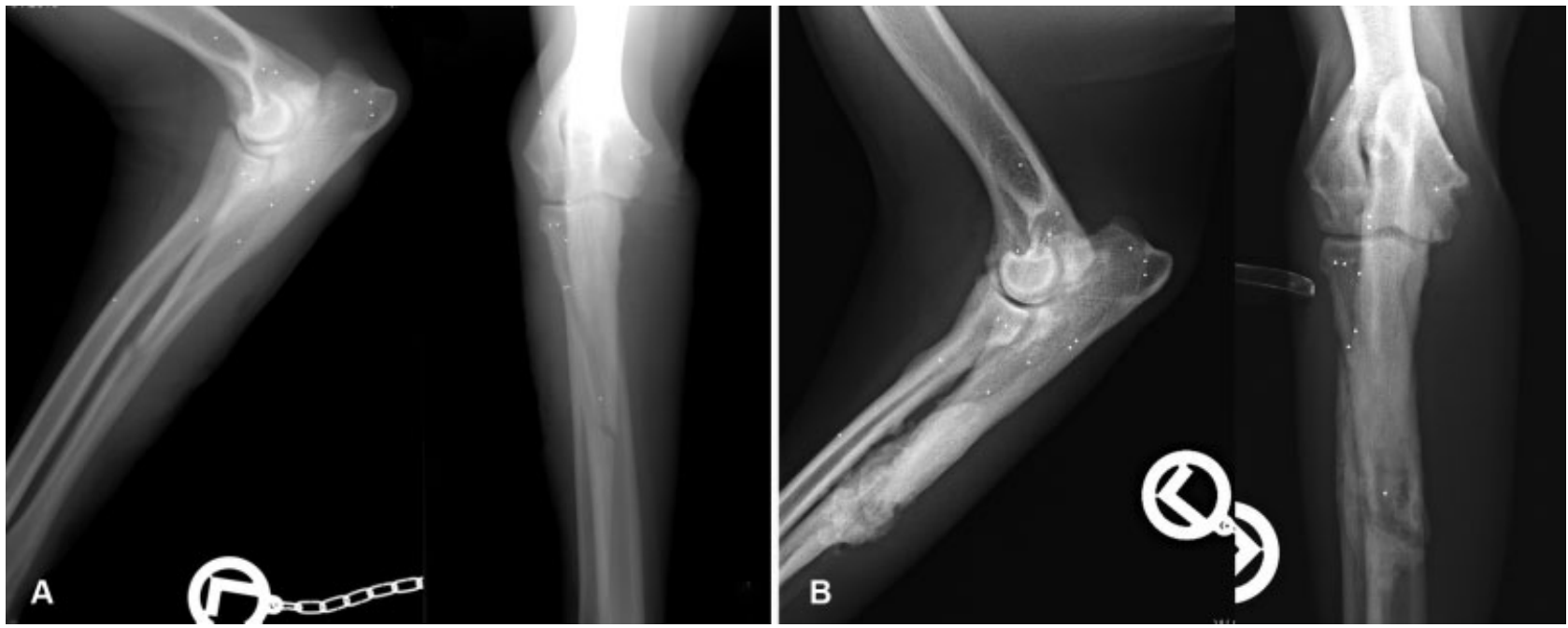

Fig. 4 Postoperative mediolateral and craniocaudal radiographic images of the left forelimb. (A) Directly after dynamic proximal ulnar osteotomy (DPUO); the bi-oblique cut in the ulna was performed in a shallow angle leading to a very long cut. Further, implanted markers are visible in the radius, ulna and humerus. (B) Twelve weeks after DPUO; callus formation at the osteotomy site is visible.

the proximal ulna segment showed the typical rotation in multiple planes as described previously, ${ }^{23}$ with cranial tipping of the segment and lowering of the MCP being the most prominent geometric changes ( $\mathbf{- F i g . ~ 5 )}$.

\section{Fluoroscopic Kinematography}

With owner's consent, three $0.8 \mathrm{~mm}$ tantalum beads (Tantalum Beads, X-Medics Scandinavia, Frederiksberg, Denmark) were implanted into ulna, radius und humerus each on the left side, immediately following arthroscopy. Transverse CT was performed after implantation using the same parameters as for the preoperative CT. Bead implantation was part of an unrelated clinical study comparing 3D elbow kinematics between sound and dysplastic elbow joints. ${ }^{10,11}$ The study was approved by the local governmental ethical committee for animal welfare (Reg. Nr.: 15-105/08).

Three weeks after implantation, fluoroscopic kinematographic gait analysis of the left elbow joint was performed. While the dog was walking on a canine treadmill (Jog A Dog, LLC, Michigan, United States), synchronized biplanar X-ray movies of the elbow joint were taken at a frame rate of 500 fps with a shutter of 0.5 milliseconds. A third synchronized high-speed video camera was used to capture a life video stream of the dog on the treadmill.

A second fluoroscopic kinematography was performed, using the identical setup 12 weeks after DPUO. By that time radiographic healing of the osteotomy had been documented and the dog showed no signs of lameness at walk and trot. To allow quantitative analysis of joint motion, it was necessary to transform the gained 2D image sequences to a 3D animation of the elbow joint. First, 3D bone models of the left humerus, ulna and radius were calculated, based on the transverse CT data acquired after bead implantation and following healing of the DPUO, respectively, using dedicated software (MeVisLab 2.0, MeVisMedical Solutions AG, Bremen, Germany). On these models, the $3 \mathrm{D}$ position of the implanted tantalum beads in respect to the local bone embedded coordinate system was digitized and stored for later use. Using special software (XrayProject; Providence, Rhode Island, United States), the image coordinates of each tantalum marker were determined

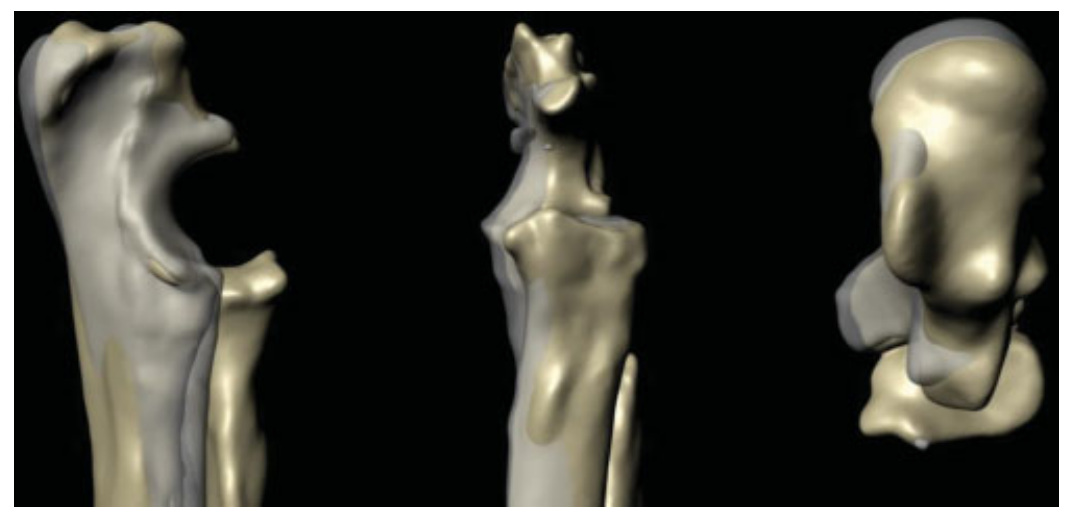

Fig. 5 Medial, cranial and dorsal view onto superimposed three-dimensional models of the radioulnar joint cup (preoperative-grey transparent; postoperative-golden). The proximal ulna segment is rotated, so the medial coronoid process is lowered and the anconeal process is pushed slightly cranioventrally towards the olecranon fossa compared with the preoperative situation. Besides the proximal ulna segment is shifted towards medial. 
manually in the synchronized X-ray movies. Tracking of bead trajectories started 30 frames before ground contact and ended 120 frames afterwards, using the life video to determine ground contact. These 120 frames represent about one-third of the whole stance phase. Restriction of the gait analysis to the first third of stance phase was due to technical reasons; during the later stance phase superimposition of the elbow joint with the thoracic body wall and/or the contra lateral limb occurred and made bead tracking unreliable. Using direct linear transformation, the $2 \mathrm{D}$ coordinates of the markers in the X-ray movies were converted to the respective $3 \mathrm{D}$ room coordinates and transferred onto the markers in the 3D models. This allowed animation of the 3D models in virtual space replicating exactly the kinematics of the elbow joint during the walk. Accuracy of this approach has been reported to be less than $0.1 \mathrm{~mm}$ and 0.1 degree. ${ }^{28}$ Based on the gained 3D animation relative axial humeroulnar rotation and relative axial radioulnar translation was quantified using defined joint coordinate systems. ${ }^{10,11}$ For measurement of relative humeroulnar motion, a 3D joint coordinate system was defined, with the z-axis orientated along a transcondylar axis of the humeral condyle, the $y$-axis parallel to the long axis of the ulna and the $x$-axis perpendicular to the first two. The medial and lateral epicondyle were used as landmarks for the transcondylar axis. Relative axial radioulnar translation was measured along a joint coordinate system with the $\mathrm{z}$-axis orientated along the caudal border of the ulna, the $x$-axis perpendicular to the z-axis and through the tip of the anconeal process and the $\mathrm{y}$-axis perpendicular to these two. Dynamic RUI was defined as any radial motion along the $\mathrm{z}$-axis in relation to the ulna. For kinematic calculations, data of three steps of the left forelimb were averaged each. Relative bone motion was expressed as maximal amplitude, measured between beginning of ground contact (30th frame) and the 150th frame. Data from three gait cycles were averaged and expressed as mean values and respective standard deviation (SD). Pairwise comparison of calculated measurements was done using a paired $t$-test.

Mean amplitude of relative axial humeroulnar rotation was 2.6 degrees (SD: 0.4 ) before and 4.5 degrees (SD: 2.0) 12 weeks after DPUO, being not significantly different $(p=0.1819)$. Therefore, rotational moment of the humerus was even increased after DPUO, with the trochlea sliding towards craniolateral driving the trochlea against the lateral aspect of the MCP. Relative axial translation between radius and ulna, representing dRUI, was $0.2 \mathrm{~mm}$ (SD: 0.03) before DPUO and $0.3 \mathrm{~mm}$ (SD: 0.08 ) afterwards (not significantly different; $p=0.1126$ ).

Based on the 3D animations, distance between the subchondral bone surface of humerus and ulna was calculated in every 10th frame and mapped onto the ulnar joint surface model, as described by Krotschek et al. ${ }^{14}$ To translate joint space width into a joint contact pattern the following definition was used: a joint space width of $\geq 1 \mathrm{~mm}$ represents no joint contact, while values $<1 \mathrm{~mm}$ equal contact. This definition is based on the known cartilage thickness of the humeral and ulnar joint surface in canine elbows. ${ }^{29,30}$ For quantitative evaluation of joint contact at the medial coronoid, the MCP was divided into nine equally sized areas using dedicated software (3-matic Research 13.0; Materialise Mimics Innovation Suite, Materialise $\mathrm{GmbH}$ Munich, Germany). First, the MCP was separated from the remaining ulnar bone model. A line was drawn along the medial aspect of the MCP; separation of the MCP was than performed along a line perpendicular to that first line transecting the lateral coronoid process (-Fig. 6A). Afterwards the joint surface of the MCP was segmented from the remaining ulnar bone (-Fig. 6B). This joint surface model was divided into nine equal parts based on its maximal bone width and length. Starting at the coronoid base three rows were numbered A, B and $C$ (from base towards tip) and three columns were numbered by 1, 2 and 3, starting at the medial aspect of the MCP (-Fig. 6C). Joint contact patterns were evaluated for the whole medial coronoid joint surface and in each of the nine parts, using ParaView (ParaView 5.6.1; Kitware Inc., Clifton Park, NY, United States). Joint contact was measured and expressed as per cent; comparison between the preoperative and postoperative situation was performed using paired $t$-test. Before DPUO, 52.5\% of the medial coronoid was in contact with the corresponding humerus and contact area was concentrated at the tip and the lateral aspect of the MCP. Following DPUO the contact area increased to $63.0 \%$ and was evenly distributed along the MCP (-Fig. 7 A, B, see additional - Video 1). Difference was significant between the

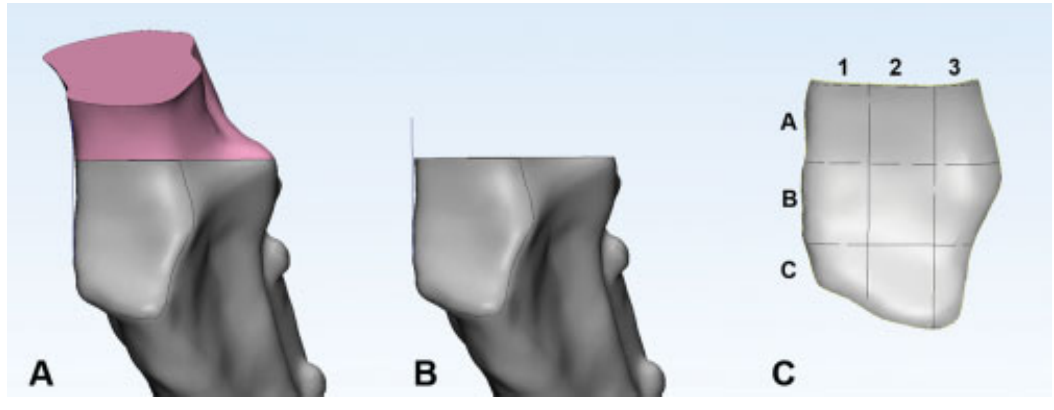

Fig. 6 Segmentation of the medial coronoid joint surface for quantification of joint contact. (A) The medial coronoid process is separated from the remaining ulnar bone by a line perpendicular to a tangent aligned with the medial border of the coronoid and transecting the lateral coronoid process. (B) Medial coronoid process after removing the ulnar bone caudally to the defined line. The joint surface is marked manually for further segmentation. (C) The segmented bone model of the medial coronoid process joint surface is further divided into nine equal parts. Width and length of each part are defined by maximum width and length of the medial coronoid process and lines are drawn perpendicular to the tangent at the medial border and the transecting line at the coronoid base respectively. 


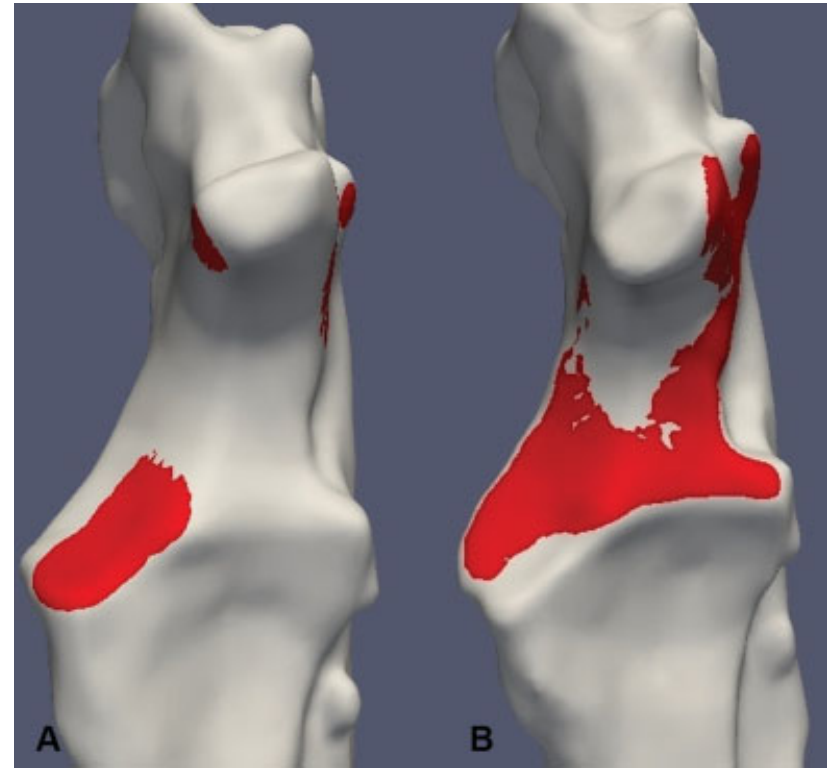

Fig. 7 joint contact patterns of the ulnar joint surface at beginning of weight bearing ( $30^{\text {th }}$ frame of animation). (A) Preoperative situation; on the left side, focal concentration of joint contact at the medial coronoid process (MCP) and slight contact at the medial and lateral aspect of the anconeal process is present. (B) Situation 12 weeks postoperative; joint contact is more homogenously distributed throughout the ulnar joint surface and craniolateral aspect of the MCP is even not in contact with the corresponding humeral trochlea.

pre- and postoperative situation $(p=0.0012)$. Comparing each of the corresponding nine parts of the MCP significant difference was found between the pre- and postoperative situation in A1 (1.1 vs. 38.3\%; $p=0.0225$ ), A2 (26.3 vs. 98.4\%; $p=0.0008$ ), A3 (18.3 vs. $72.6 \% ; p=0.0261$ ) and C3 (95.9 vs. $27.9 \% ; p=0.0071)$. No significant difference was found in the other compartments of the medial coronoid.

\section{Video 1}

Video shows animation of humeroulnar joint contact patterns preoperatively and following dynamic proximal ulnar osteotomy. Red areas represent contact area, defined as a joint space width of $1 \mathrm{~mm}$ or less between the humeral and ulnar joint surface. Online content including video sequences viewable at: https://www.thieme-connect.com/products/ ejournals/html/10.1055/s-0039-1698440.

\section{Discussion}

As previously described, DPUO did not result in complete resolution of axial sRUI. ${ }^{23}$ Likewise, dRUI under load remained unaffected by DPUO. Therefore, it seems unlikely that any mechanical and/or clinical improvement following DPUO $^{13-15}$ would only be related to changes in RUI. In the present case, beneficial effects might be attributed to the fact that the humeroulnar joint contact area was increased (52.5 vs. $63.0 \%$ ) and became more evenly distributed over the area of the MCP following DPUO. In particular, the coronoid base showed significantly increased contact (A1-A3 compartments), while the lateral aspect of the coronoid tip was unloaded following DPUO (C3: 95.9 preoperatively vs. 27.9\% postoperatively). Compared with the preoperative concentration of a relatively small contact area at the lateral aspect of the MCP, which corresponds well with the typical topography of early MCPD lesions, ${ }^{31}$ we interpret this change in contact pattern to be a biomechanical improvement in overall humeroulnar joint mechanics, and we would expect a beneficial clinical effect. This assumption is strengthened by the observation during second-look arthroscopy following DPUO in joints with medial compartment disease, documenting fibrocartilage growth at the formerly denuded joint surfaces. ${ }^{32}$ Second-look arthroscopy was not available in the present case. Further, changes of the joint contact area within the humeroradial joint were not evaluated. Thus, we are not able to describe overall changes of the contact pattern within this canine elbow after DPUO.

After healing of the osteotomy, the dog showed no detectable lameness and was pain free on elbow palpation. Owners reported good function as well as no signs of lameness and pain. Sixteen months after DPUO, according to the owners, the dog still showed no signs of pain or lameness, even after intense exercise. However, because force plate analysis was not available at any point in time, it is impossible to objectivize the effect of DPUO on functional outcome.

The focal concentration of humeroulnar joint contact, seen before DPUO, is probably the result of humeral rotational instability present in elbow joints with MCPD. ${ }^{11}$ Comparing sound and dysplastic elbow joints revealed that in affected elbows the humeral condyle rotates externally with the humeral trochlea being driven against the lateral part of the MCP. ${ }^{11}$ This motion pattern was also present in our case, and even markedly increased after DPUO. Although the anconeal process was pushed deeper into the olecranon fossa following DPUO, this did not constrain axial humeroulnar rotation. We speculate that the complex 3D deformity of the proximal ulna, induced by DPUO, ${ }^{23}$ allows more axial rotation, because the MCP rotates distally and therefore there is less rotational restraint for the trochlea. When comparing the deformity of the ulna in the present case to what has been reported previously following DPUO, ${ }^{23,27}$ it seems that malalignment was less severe than what typically occurs. This might be due to the very long bi-oblique cut in the ulna, which exceeded the recommendations made by Caron and Fitzpatrick, ${ }^{27}$ allowing less movement to the proximal ulna segment following the osteotomy.

\section{Conclusion}

To our knowledge this is the first report of in vivo joint kinematics and joint contact pattern following DPUO in a canine elbow joint with MCPD. Although DPUO did not restore normal radioulnar congruence while potentially increasing rotational humeroulnar instability, the 
humeroulnar joint contact became more homogenously distributed at the MCP following DPUO. We speculate that this biomechanical change corresponds with the functional improvement in this patient.

\section{Author Contributions}

All authors contributed to conception of study, study design, acquisition of data and data analysis and interpretation. All authors drafted, revised and approved the submitted manuscript.

\section{Conflicts of interest}

None declared.

\section{References}

1 Cook JL. Forelimb lameness in the young patient. Vet Clin North Am Small Anim Pract 2001;31(01):55-83

2 Fitzpatrick N, Smith TJ, Evans RB, Yeadon R. Radiographic and arthroscopic findings in the elbow joints of 263 dogs with medial coronoid disease. Vet Surg 2009;38(02):213-223

3 Gemmill TJ, Clements DN. Fragmented coronoid process in the dog: is there a role for incongruency? J Small Anim Pract 2007;48 (07):361-368

4 Kirberger RM, Fourie SL. Elbow dysplasia in the dog: pathophysiology, diagnosis and control. J S Afr Vet Assoc 1998;69(02): 43-54

5 Puccio M, Marino DJ, Stefanacci JD, McKenna B. Clinical evaluation and long-term follow-up of dogs having coronoidectomy for elbow incongruity. J Am Anim Hosp Assoc 2003;39(05):473-478

6 Krotscheck U, Kalafut S, Meloni G, et al. Effect of ulnar ostectomy on intra-articular pressure mapping and contact mechanics of the congruent and incongruent canine elbow ex vivo. Vet Surg 2014; 43(03):339-346

7 McConkey MJ, Valenzano DM, Wei A, et al. Effect of the proximal abducting ulnar osteotomy on intra-articular pressure distribution and contact mechanics of congruent and incongruent canine elbows ex vivo. Vet Surg 2016;45(03):347-355

8 Eljack H, Böttcher P. Relationship between axial radioulnar incongruence with cartilage damage in dogs with medial coronoid disease. Vet Surg 2015;44(02):174-179

9 Samoy Y, Van Ryssen B, Gielen I, Walschot N, van Bree H. Review of the literature: elbow incongruity in the dog. Vet Comp Orthop Traumatol 2006;19(01):1-8

10 Rohwedder T, Fischer M, Böttcher P. In vivo fluoroscopic kinematography of dynamic radio-ulnar incongruence in dogs. Open Vet J 2017;7(03):221-228

11 Rohwedder T, Fischer M, Böttcher P. In vivo axial humero-ulnar rotation in normal and dysplastic canine elbow joints. Tierarztl Prax Ausg K Klientiere Heimtiere 2018;46(02):83-89

12 Fitzpatrick N, Garcia TC, Daryani A, Bertran J, Watari S, Hayashi K. Micro-CT structural analysis of the canine medial coronoid disease. Vet Surg 2016;45(03):336-346

13 Fitzpatrick N, Yeadon R. Working algorithm for treatment decision making for developmental disease of the medial compartment of the elbow in dogs. Vet Surg 2009;38(02):285-300

14 Krotscheck U, Böttcher PB, Thompson MS, Todhunter RJ, Mohammed HO. Cubital subchondral joint space width and CT osteoabsorptiometry in dogs with and without fragmented medial coronoid process. Vet Surg 2014;43(03):330-338
15 Starke A, Böttcher P, Pfeil I. Radiologic quantification of the elbow conformation with a new method for acquiring standardized $\mathrm{x}$ rays under load. Reference values for medium sized and large dogs without dysplasia of the elbow joint [article in German]. Tierarztl Prax Ausg K Klientiere Heimtiere 2013;41(03):145-154

16 Starke A, Böttcher P, Pfeil I. [Comparative radiologic examination of the canine elbow with and without elbow dysplasia under standardized load]. Tierarztl Prax Ausg K Klientiere Heimtiere 2014;42(03):141-150

17 Lau SF, Hazewinkel HA, Grinwis GC, et al. Delayed endochondral ossification in early medial coronoid disease (MCD): a morphological and immunohistochemical evaluation in growing Labrador retrievers. Vet J 2013;197(03):731-738

18 Lau SF, Wolschrijn CF, Hazewinkel HA, Siebelt M, Voorhout G. The early development of medial coronoid disease in growing Labrador retrievers: radiographic, computed tomographic, necropsy and micro-computed tomographic findings. Vet J 2013;197(03):724-730

19 Griffon D. Surgical diseases of the elbow. In: Tobias KM, Johnston SA, eds. Veterinary Surgery: Small Animal. St. Louis: Elsevier; 2012:724-759

20 Gilson SD, Piermattei DL, Schwarz PD. Treatment of humeroulnar subluxation with a dynamic proximal ulnar osteotomy. A review of 13 cases. Vet Surg 1989;18(02):114-122

21 Preston CA, Schulz KS, Taylor KT, Kass PH, Hagan CE, Stover SM. In vitro experimental study of the effect of radial shortening and ulnar ostectomy on contact patterns in the elbow joint of dogs. Am J Vet Res 2001;62(10):1548-1556

22 Ness MG. Treatment of fragmented coronoid process in young dogs by proximal ulnar osteotomy. J Small Anim Pract 1998;39 (01):15-18

23 Böttcher P, Bräuer S, Werner H. Estimation of joint incongruence in dysplastic canine elbows before and after dynamic proximal ulnar osteotomy. Vet Surg 2013;42(04):371-376

24 Quinn MM, Keuler NS, Lu Y, Faria ML, Muir P, Markel MD. Evaluation of agreement between numerical rating scales, visual analogue scoring scales, and force plate gait analysis in dogs. Vet Surg 2007;36(04):360-367

25 Ohlerth S, Tellhelm B, Amort K, et al. Explanation of the IEWG grading system. 30th annual meeting of the International Elbow Working Group; 2016; Vienna, Austria

26 Eljack H, Werner H, Böttcher P. Sensitivity and specificity of 3D models of the radioulnar joint cup in combination with a sphere fitted to the ulnar trochlear notch for estimation of radioulnar incongruence in vitro. Vet Surg 2013;42(04):365-370

27 Caron A, Fitzpatrick N. Bi-oblique dynamic proximal ulnar osteotomy: surgical technique and clinical outcome in $86 \mathrm{dogs}$. Vet Surg 2016;45(03):356-363

28 Brainerd EL, Baier DB, Gatesy SM, et al. X-ray reconstruction of moving morphology (XROMM): precision, accuracy and applications in comparative biomechanics research. J Exp Zool A Ecol Genet Physiol 2010;313(05):262-279

29 Zeissler M, Maierl J, Grevel V, Oechtering G, Böttcher P. Cartilage thickness and split-line pattern at the canine humeral trochlea. Vet Comp Orthop Traumatol 2010;23(05):343-347

30 Gendler A, Keuler NS, Schaefer SL. Computed tomographic arthrography of the normal canine elbow. Vet Radiol Ultrasound 2015;56(02):144-152

31 Temwichitr J, Leegwater PA, Hazewinkel HA. Fragmented coronoid process in the dog: a heritable disease. Vet J 2010;185(02):123-129

32 Olivieri M. Dynamic proximal ulnar osteotomy. Proceedings, 22nd Annual Scientific Meeting of the European College of Veterinary Surgeons; 2013; Rome, Italy 